\title{
A Highly Efficient Culture Technique for Derivation of Motor Neurons from Human Umbilical Cord Derived Mesenchymal Stem Cells
}

\author{
Shetty $\mathrm{P}^{*}$, Pradhan S and Viswanathan $\mathrm{C}$
}

Regenerative Medicine Group, Reliance Life Sciences Pvt. Navi Mumbai, India

*Corresponding author: Shetty P, Regenerative Medicine Group, Reliance Life Sciences Pvt Ltd., Navi Mumbai 400 701, India, Fax: 091 2240678099, Tel: 091 2240678352, E-mail: prathibha.shetty@relbio.com

Citation: Shetty P, Pradhan S, Viswanathan C (2015) A Highly Efficient Culture Technique for Derivation of Motor Neurons from Human Umbilical Cord Derived Mesenchymal Stem Cells. J Neurol Neurol Disord 2(1): 101. doi: 10.15744/2454-4981.2.101

Received Date: December 19, 2014 Accepted Date: March 23, 2015 Published Date: March 27, 2015

\begin{abstract}
Motor Neuron Diseases (MND) are a group of progressive neurological disorders that destroy motor neurons, cells that control essential muscle activity. Despite the advances in treatment modalities, the overall survival rate has not changed for decades. This is mainly due to the lack of effective methods. There is only one drug, Riluzole, as of date, approved by US FDA which only prolongs the life span by few months. Therefore, it is necessary to develop other cell based methods as a novel therapy for improving the outcome of the disease. Hence, an in vitro study has been carried out on the differentiation of human mesenchymal stem cells (hMSCs) to motor neurons. The knowledge of key molecules involved in the process of differentiation is important for efficient differentiation. In the present study, umbilical cord derived MSCs (UC-MSCs) were differentiated to mature motor neurons in vitro. Here, we have developed an efficient culture system for derivation of motor neurons (MN) from UC-MSCs. This method of induction led to higher yield of motor neuron markers. The differentiated cells exhibited typical motor neuron like morphology and characterization studies revealed that the differentiated cells showed high expression of motor neuron markers such as HB9 and Olig 2. Highly efficient $73.57 \%$ AChT ${ }^{+}$ motor neuron like cells were obtained. The protocol for differentiation was found to be effective in differentiating MSCs to MNs. Together these findings demonstrate the feasibility of using MSC derived motor neuron like cells in regenerative medicine applications and provides a ray of hope for MND patients.

Keywords: Human umbilical cord; Mesenchymal stem cells; Embryoid like bodies; Neurospheres; Motor neuron; Neurodegenerative diseases

Abbreviations: UCMSCs-Umbilical cord Mesenchymal stem Cells; MNs-Motor neurons; hESCs-human Embryonic Stem Cells; iPSCs-induced Pluripotent Stem Cells; AChT-Choline acetyl transferase
\end{abstract}

\section{Introduction}

Motor neurons (MNs) are a specialized class of neurons that reside in the brain and spinal cord and project axons from central nervous system in organized and discrete patterns to muscles to control their activity. They gather and transmit electrochemical signals over long distance from the central nervous system to the outer parts of our body [1]. MNDs are progressive neurological disorders which destroy upper motor neurons and lower motor neurons from brain and spinal cord [2]. Motor neuron diseases can be classified into various types such as Amyotrophic Lateral Sclerosis (ALS), Spinal muscular atrophy (SMA), progressive bulbar palsy (PBP) and primary lateral sclerosis (PLS). In each type of disease, motor neurons of different areas of CNS are affected. Current treatment options are directed towards alleviating the symptoms and therapies to slow down disease progression. The only drug licensed by US FDA for the treatment of MND is riluzole, which was designed as a specific glutamate antagonist. The incidence of people who are likely to develop MND each year is about two people in every 100,000. The prevalence or number of people living with MND at any one time is approximately seven in every 100,000 [3].

Stem cell transplantation is a new way to improve the patients' conditions and reduce the period of disabilities. Considerable progress has been made in the past decade in differentiating human pluripotent stem cells like hESCs and iPSCs into MNs by various scientists using a cocktail of different growth factors. A number of protocols have been developed, which utilize various steps, for differentiation of ESC and iPSC to motor neurons. In 2002, for the first time hESCs were differentiated to Motor Neurons by Wichterle $\mathrm{H}$. et al. [4]. They identified the inductive signals and transcription factors in MN generation. Hu et al. group [5] efficiently differentiated human embryonic stem cells (hESCs) to neural epithelial cells and then to functional spinal motor neurons. Recently, Qu. Q et al. [1] has also differentiated hESCs and hiPSCs to motor neurons. This protocol was shown to be equally effective in generating motor neurons from human induced pluripotent stem (iPS) cells. In 2010, Rossi S. et al. [6] transplanted spinal cord injured animals with a high purity population of human motor neuron progenitors (hMNP) derived from human embryonic stem cells (hESCs). 
Despite these encouraging advances, limitations remain in the existing protocols that hamper the use of human pluripotent stem cells for therapeutic purpose. MSCs have shown great promise in cell and gene therapy applications, because of their multipotentiality and capacity for extensive self-renewal. These applications demonstrate the plasticity of these adult stem cells and their usefulness in multiple tissue repair and regeneration and in cell therapy applications [7].

Park H. et al. [8] genetically engineered hBM-MSCs to express MN associated transcription factors, Olig2 and Hb9, and then treated the hMSCs expressing Olig2 and Hb9 with optimal MN induction medium (MNIM). These cells were able to form functional connections with muscle fibers in vitro and when transplanted into an injured organotypic rat spinal cord slice culture, an ex vivo model of spinal cord injury, they exhibited characteristics of MNs. Liu et al. [9] has generated MN like cells from UCMSCs. The results showed that heparin combined with basic fibroblast growth factor (bFGF) promoted the differentiation of umbilical cord mesenchymal stem cells into motor neuron-like cells but with low efficiency.

Selection of suitable growth factors for differentiation and inducing the cells with these growth factors at appropriate time points is the first step towards efficient differentiation. The aim of this study was to differentiate UC-MSCs into motor neurons using a novel protocol and characterization of the cells at the molecular and cellular level. Our protocol to differentiate UCMSCs to motor neurons is an attempt to use cells which could be more effective in the treatment of neurodegenerative disorders. Here we develop a method for highly efficient ( $73 \%$ ) derivation of mature MNs from human adult stem cells. In summary, this technique efficiently differentiates UC-MSCs into mature motor neurons that can be used for preclinical and clinical studies. This study should facilitate the therapeutic applications of human MNs for the treatment of MND in future.

\section{Materials and Methods}

\section{Culturing and expansion of UC-MSCs}

Umbilical cord derived MSCs (UC-MSCs) were isolated from umbilical cord tissue obtained by explant method. Further these cells were expanded and characterized for the presence of MSC markers like CD73 and CD105 as per ISCT (International Society for Cellular Therapy) criteria and were found to be $\geq 90 \%$ positive [10]. The cord tissue was obtained after proper consenting process and approvals from IC-SCR (Institutional Committee for Stem Cell Research).

Our method is based on embryoid body (EB) induction, neurosphere formation followed by neural rosette formation and motor neuron maturation. UC-MSCs, Passage 2 were expanded in MSC expansion medium containing DMEM: F12 (LonzaTM, Switzerland), 10\% FBS (HyClone ${ }^{\varpi}$, USA) and 2 ng/ml bFGF (Sigma ${ }^{\oplus}$, USA). These flasks were harvested at 80-90\% confluency.

\section{Formation of cell aggregates}

For the formation of cell aggregates, $0.8 \times 10^{6}$ cells were plated in each well of low adherent six well plates in MSC expansion medium to obtain embryoid bodies like aggregates. Cell aggregates were formed on Day 3. Media was changed to neurosphere medium. Neurosphere media consists of 1X N2, 1X B27 (Gibco ${ }^{\oplus}$, USA), 5 ng/ml bFGF and 1X sodium-pyruvate (Gibco ${ }^{\varpi}$ ). On day 7 , when the cells formed dense aggregates or neurospheres in suspension, they were plated on laminin coated flasks. Few neurospheres and EB like aggregates were separated and used for characterization studies.

\section{Neuronal differentiation of MSCs}

To induce neuronal differentiation, T-25 flasks were coated with Laminin (Sigma ${ }^{\oplus}$ ). 0.1\% laminin was diluted in $1 \mathrm{X}$ DPBS (Dulbecco's Phosphate Buffer Saline, Gibco ${ }^{\circ}$ ). Laminin coating solution was added to each flask and incubated at $37^{\circ} \mathrm{C}$ for 2 hrs. Neurospheres obtained by the above method, were plated on Laminin coated T-25 flasks in which Media 1 (DMEM: F12, 10\% FBS and $1 \mathrm{mM} \beta$-mercaptoethanol (Sigma $\left.{ }^{\oplus}\right)$ ) was added. On day 3 after plating, $2 \mathrm{mM}$ Beta mercaptoethanol was added to each flask for a period of 3 hours. The media was then substituted by Media 2 containing DMEM: F12, $1 \mu$ M Retinoic acid (Sigma $\left.{ }^{\circledR}\right)$ and $5 \mu \mathrm{M}$ Forskolin $\left(\right.$ Sigma $\left.^{\circledR}\right)$. After 4 days, with a day of media change, media was changed to Media 3 consisting of DMEM: F12, $1 \mu \mathrm{M}$ Retinoic acid and $5 \mu \mathrm{M}$ Forskolin, $5 \mu \mathrm{g} / \mathrm{ml}$ Heparin (LobaChemie), India, $10 \mathrm{ng} / \mathrm{ml} \mathrm{bFGF}$. After 6-8 days, with alternate day media change, media was changed to Media 4 (DMEM: F12, 100ng/ml SHH R \& D Systems ${ }^{\varpi}$, USA), $1 \mu$ M Retinoic acid and $5 \mu$ M Forskolin, $5 \mu \mathrm{g} / \mathrm{ml}$ Heparin, $10 \mathrm{ng} / \mathrm{ml} \mathrm{bFGF}$ ). This protocol was a 35 day protocol which involved monitoring for morphological changes at regular intervals.

The cells at different stages of differentiation were classified as follows:

\begin{tabular}{|c|c|}
\hline Stage I & Undifferentiated UC-MSCs \\
\hline Stage II & Embryoid Body like aggregates \\
\hline Stage III & Neurospheres \\
\hline Stage IV & Motor Neuron Progenitors (MNP) \\
\hline Stage V & Mature Motor Neurons \\
\hline
\end{tabular}

\section{Characterization Studies}

Cells of all the stages (Stage I - Stage V) were taken for characterization. Molecular characterization was performed using RT-PCR and Real Time PCR. Cellular characterization was done using Flow Cytometry and Immunocytochemistry. 


\section{RT-PCR analysis}

Cells were harvested at each stage and cell pellet was collected and stored at $-20^{\circ} \mathrm{C}$. RNA was isolated from cell pellet using RNA isolation kit (RNeasy Micro Kit Qiagen). cDNA was synthesized using cDNA synthesis kit (Superscript II First strand cDNA synthesis kit from Invitrogen). AB gene (2x) master mix was used. PCR mix contains cDNA sample, forward and reverse primer and nuclease-free water to a final volume of $12.5 \mu \mathrm{l}$. Cycling parameters were as follows: denaturation at $94{ }^{\circ} \mathrm{C}$ for 45 seconds, annealing for 45 seconds, and elongation at $72{ }^{\circ} \mathrm{C}$ for 1 minute, for $30 \mathrm{cycles}$ and final extension at $72{ }^{\circ} \mathrm{C}$ for 10 minutes on Biorad Thermal Cycler.

RT-PCR was done to amplify marker genes under study (Table 1). Primers were provided by Sigma-Aldrich. Agarose gel electrophoresis was performed. The agarose gel was run at a constant voltage of $100 \mathrm{~V}$ for $\sim 1$ hour till the ladder was resolved and orange dye had migrated more than two-thirds of the length of the gel. Bands were visualized by exposure to UV light and gels were photographed using Pharmacia Biotech Gel Documentation System.

\begin{tabular}{|c|c|c|c|}
\hline Gene Name & $\begin{array}{l}\text { Annealing } \\
\text { Temperature }\end{array}$ & Primer sequence & Expected Product Size (bp) \\
\hline GAPDH & $60{ }^{\circ} \mathrm{C}$ & $\begin{array}{l}\text { 5'-TGAAGGTCGGAGTCAACGGATTTGGT-3' } \\
\text { 5'-CATGTGGGCCATGAGGTCCACCAC-3' }\end{array}$ & 890 \\
\hline Oct3/4 & $73.2^{\circ} \mathrm{C}$ & $\begin{array}{l}\text { 5'-CGRGAAGCTGGAGAAGGAGAAGCTC-3' } \\
\text { 5'-CAAGGGCCGCAGCTTACACATGTTC-3' }\end{array}$ & 247 \\
\hline Nanog & $69.2^{\circ} \mathrm{C}$ & $\begin{array}{l}\text { 5'-CCTCCTCCATGGATCTGCTTATTCA-3' } \\
\text { 5'-CAGGTCTTCACCTGTTTGTAGCTGAG-3' }\end{array}$ & 262 \\
\hline Nestin & $61.5^{\circ} \mathrm{C}$ & $\begin{array}{l}\text { 5'-TTTTCCACTCCAGCCATCC-3' } \\
\text { 5'-CCAGAAACTCAAGCACCAC-3' }\end{array}$ & 395 \\
\hline B-tubulin & $55.9^{\circ} \mathrm{C}$ & $\begin{array}{c}\text { 5'-CTTACTACTGTTAGATCCCAGGAAT-3' } \\
\text { 5'-TGAGACGATGTCCTCCATA-3' }\end{array}$ & 240 \\
\hline NF-M & $62.9^{\circ} \mathrm{C}$ & $\begin{array}{l}\text { 5'-GAGCGCAAAGACTACCTGAAG-3' } \\
\text { 5'-CAGCGATTTCTAATCCAGAGC-3' }\end{array}$ & 400 \\
\hline Nkx 2.2 & $60{ }^{\circ} \mathrm{C}$ & $\begin{array}{l}\text { 5'-GAGTTGACTCTCGGCTCCAC-3' } \\
\text { 5'-AACCCAAACAAGCCACAAAG-3' }\end{array}$ & 527 \\
\hline Olig 2 & $60^{\circ} \mathrm{C}$ & $\begin{array}{l}\text { 5’-CCTTGCTCCTCTTCСТCСТT-3' } \\
\text { 5'-ACCCAAACTGTTTCCACAGC-3' }\end{array}$ & 503 \\
\hline Hb9 & $57^{\circ} \mathrm{C}$ & $\begin{array}{l}\text { 5'-GCAGGCGGCGCTCTAC-3' } \\
\text { 5'-TTCCCCAGGAGGTTCGAC-3' }\end{array}$ & 164 \\
\hline AChT & $56^{\circ} \mathrm{C}$ & $\begin{array}{l}\text { 5'-TCATTAATTTCCGCCGTCTC-3' } \\
\text { 5'-AGTCCCGGTTGGTGGAGTC-3' }\end{array}$ & 188 \\
\hline
\end{tabular}

Table 1: The sequences of the primers used to amplify marker genes

\section{Relative gene expression studies by qRT-PCR}

For quantification of the neuronal genes, cDNA from the undifferentiated and differentiated cells were amplified using Taqman Universal PCR Master Mix (ABI, USA) in Sequence Detection Systems. Taqman MGB probes with 50 FAM $^{\circledR}$ dye label were used for amplifying the gene. The Taqman Gene Expression Assays were from Applied Biosystems. One microgram of the cDNA was subjected to qRT-PCR using specific primer and probe for Nestin (Hs00707120_s1) $\beta$-tubulin (Hs00742828_s1), Hb9 (Hs00252848_s1), AChT (Hs00907365_s1) and 18sRNA (Hs99999901_s1). Cycling parameters were as follows: initial step at 95 ${ }^{\circ} \mathrm{C}$ for $10 \mathrm{~min}$, denaturation at $92{ }^{\circ} \mathrm{C}$ for $15 \mathrm{~s}$, annealing at $60{ }^{\circ} \mathrm{C}$ for $1 \mathrm{~min}$. Denaturation and annealing steps were repeated for 40 cycles. Analysis of the amplified products was by fluorescence detection using Real time ABI system SDS 7500 version 1.2 .2 software. The amount of transcript was normalized to an internal 18s RNA control and averaged from triplicate samples.

\section{Flow cytometric analysis}

Immunophenotyping of the undifferentiated and differentiated cells was done by flow cytometer. Embryoid Bodies like aggregates/Neurospheres/ cells at stage IV and V were harvested using TrypLE Select. Cells were fixed using Cytoperm/Cytofix (BD Pharmingen ${ }^{\mathrm{TM}}$ ) was at $4{ }^{\circ} \mathrm{C}$ for $30 \mathrm{~min}$. Harvested adherent cells were washed with $1 \mathrm{X}$ DPBS, and 20,000 cells were used to follow cell surface antigen expression. Cells at all the stages were assessed for the expression of undifferentiated stem cell markers like Oct 4 (BD PharmingenTM) and Nanog $\left(\right.$ Chemicon $\left.^{\varpi}\right)$, primitive neuronal markers such as Pax 6, Sox 1 (Abcam $\left.{ }^{\circledR}\right)$, $\beta$-tubulin and Nestin (Chemicon ${ }^{\circledR}$ ) and motor neuron specific markers including Islet-1(R \& D Systems $\left.{ }^{\circledR}\right)$, MAP-2 (Chemicon $\left.{ }^{\circledR}\right)$, Olig2 $\left(\right.$ Abcam $\left.^{\circledast}\right), \mathrm{Hb} 9\left(\right.$ Bioss $\left.^{\circledast}\right)$ and Acetyl Choline Transferase (Chemicon $\left.{ }^{\circledast}\right)$. Appropriate isotype and secondary antibody controls were used during the assay. These cells were analyzed with an FACS Calibur Flow Cytometer (FACS Calibur, BD, USA) equipped with a $488 \mathrm{~nm}$ Argon Laser. Approximately 10000 events were acquired and analyzed with Cell Quest Software. 


\section{Immunofluorescence}

Cells were washed once with cold PBS, fixed with 4\% paraformaldehyde for 10 min, permeabilized with $0.1 \%$ Triton X-100 in PBS and blocked with BSA. Primary antibodies such as, Islet-1 (R \& D Systems ${ }^{\circledR}$ ), Nestin, $\beta$-tubulin, Neu N, MAP-2 , AChT (Chemi$\operatorname{con}^{\otimes}$ ), Olig 2, Hb9 (Bioss ${ }^{\circledast}$ ) were incubated with samples overnight at $4^{\circ} \mathrm{C}$, followed by fluorescein isothiocyanate (FITC), Alexa Flour 488, Alexa Flour 568, Alexa Flour 546-conjugated secondary antibody (BD Pharmingen ${ }^{\mathrm{TM}}$ ). Nuclei were counterstained with DAPI (4', 6-diamidino-2-phenylindole; Sigma). Cells were double stained with various markers labeled with different fluorochromes raised in different species. Images were collected with Axiovert 200M microscope (Zeiss).

\section{Results}

\section{Morphological Characterization}

MSCs from the human umbilical cord tissue samples were derived by the explant culture method as described earlier by Shetty et al. (2010). The expanded UC-MSCs fulfilled the criteria for their markers as set by ISCT. These were spindle shaped and had fibroblast like morphology as shown in Figure 1A.

\section{Formation of cell aggregates}

In our induction protocol, we first induced the cells to form EB like aggregates and further to neurospheres. Embryoid bodies like aggregates were seen as aggregates of cells in 2 days (Figure 1B). Neurospheres were then formed from EBs in 3 days as compact bodies when compared to the latter. The EBs and neurospheres were characterized for the marker expression by molecular and cellular methods.

\section{Neuronal differentiation}

Our modified protocol was initially reported by Park et al, 2012 who used genetically engineered hMSCs for differentiation. The neurospheres were then plated on laminin coated flasks (Figure 1C) and cultured in Media 1 in which consists of $\beta$-mercaptoethanol and $10 \%$ FBS. After 48 hours in culture, 2mM Beta-mercaptoethanol was added to the flasks. After 3 hours, media was changed to media 2. Media 2 consists of Retinoic Acid (RA) and Forskolin. 24 hours after media change, many shiny refractile bodies were seen in the culture. After 4 days in culture and one media change, the media was replaced with media 3 . At the end of $7^{\text {th }}$ day the number of shiny cells in the culture increased. Within 24 hours, shiny colony structure became more distinct forming neural rosette. All the rosettes had compact shiny center and sharp edges as shown in the Figure 1D. Before media change, some cells were taken for characterization (stage IV). After 6-8 days in culture and 2-3 media changes, media was changed to media 4 . SHH is additionally present in Media 4. RA and SHH required to regulate the expression of homeodomain and basic helix-loop helix transcription factors, which designate the specification of MNs in the ventral spinal cord [8]. Stage IV cells or MN progenitors are shiny bodies in the culture, some of which were bipolar in nature (Figure 1D). Mature motor neurons (stage V) cells have a typical neuronal morphology with shiny nucleus, retracted cytoplasm and extended processes (Figure 1E, F).
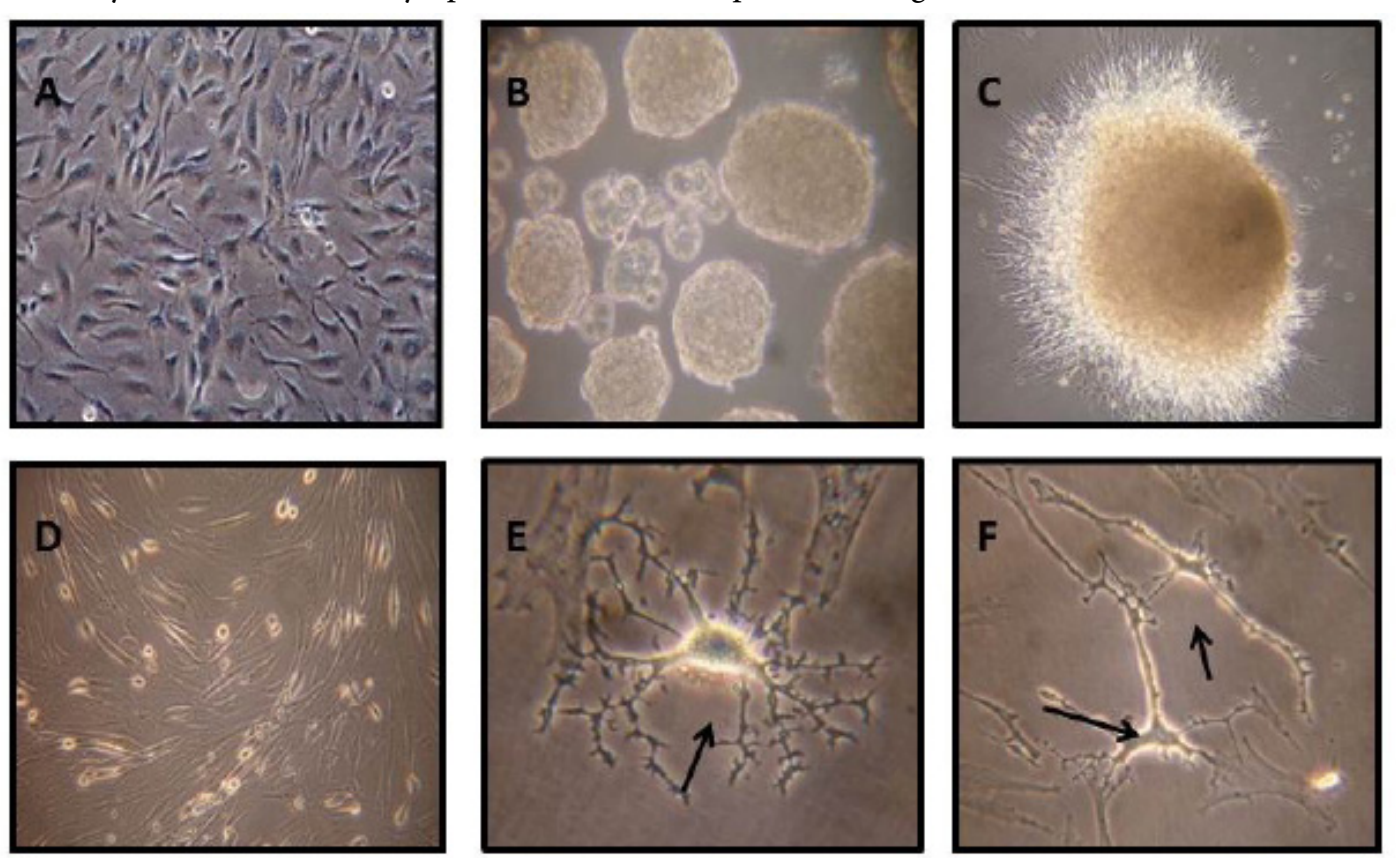

Figure 1: Morphology of MNs at different stages of differentiation

A: Morphology of undifferentiated UC-MSCs (Stage I); B: Embryoid Bodies like aggregates formed on low adherent plates (Stage II); C: Neurospheres plated on laminin coated flasks (Stage III) where cells have started migrating from the neurosphere; D: Initiation of Motor Neuron Progenitors (Stage IV), many shiny refractile bodies can be seen in the culture; E: Arrow indicates the morphology of differentiated cells with a shiny refractile nucleus (Stage V); F: Arrows indicate mature MN like cells which exhibit multi processes arising from the cells and forming connections 


\section{Molecular Characterization}

Gene expression analysis of differentiated MSCs by RT-PCR and qRT-PCR: Cells of all the stages (Stage I - Stage V) were characterized using RT-PCR. cDNA was checked for expression of various markers such as Oct 4, Nanog, Nestin, $\beta$-tubulin, NF-M, Nkx 2.2, Olig2, Hb9, AChT and GAPDH. As shown in Figure 2, cells express different markers at different stages. Human Brain cDNA was used as a positive control. The expression of Oct 4 and Nanog was reduced from Stage I to Stage V. The expression of nestin, $\beta$-tubulin was observed in the final stages (stage IV and V) of differentiation. The expression of NF-M which is a Neuronal marker seems to be equal in all the stages. Nkx 2.2 which is involved in morphogenesis of central nervous system has an increased expression in neurosphere stage. Olig 2 and $\mathrm{Hb} 9$ which are motor neuron specific markers are increased in Stage V as compared to the previous stages. The densitometric analysis of the RT-PCR showed higher intensities of the motor neuron specific genes such as Olig 2, Hb9 and AChT. The differentiated cells showed a very high intensity of expression for the above mentioned genes. The intensity of expression was found to be greater than $70 \%$ as compared to the undifferentiated. The expression of AChT in Stage V increased remarkably as compared to all the previous stages.

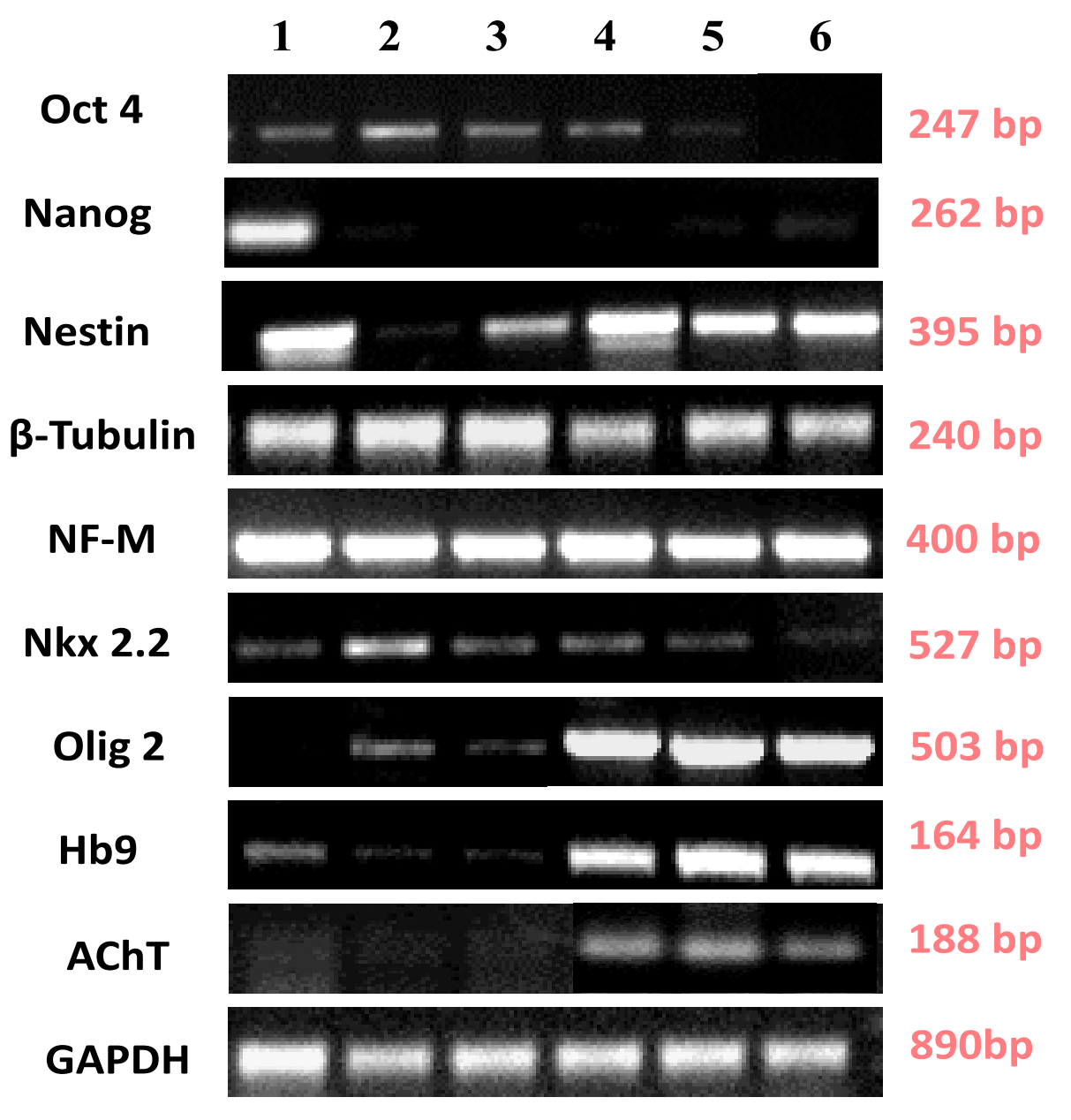

Figure 2: RT-PCR analysis showing expression of various markers at genetic level; motor neuron markers being upregulated at stage IV and V, highest expression is at stage $\mathrm{V}$.

LANE 1: Undifferentiated UC-MSCs

LANE 2: Embryoid Bodies like aggregates

LANE 3: Neurospheres

LANE 4: Motor neuron progenitors (Stage IV)

LANE 5: Motor neuron like cells (StageV)

LANE 6: Human Brain cDNA (control)

Gene expression analysis of differentiated MSCs by qRT-PCR: qRT-PCR analysis was carried out of genetic markers such as Nestin, $\beta$-tubulin, NF-H, Hb9 and Acetyl Choline Transferase. UCMSCs differentiated specifically towards the motor neurons in the presence of specific growth factors used in protocol. It was observed that in the cells of stage V, Nestin and $\beta$-tubulin genes were downregulated as compared to stage IV, whereas the expression of $\mathrm{Hb} 9$ and AChT found to be was upregulated. They showed a 2 fold increase in the expression at stage V as compared to 1.5 fold in stage IV (Figure 3). Expression of these motor neuronspecific genes was clearly upregulated in the later stages, which suggests that UCMSCs derived from a foetal tissue, are conducive to differentiate along the motor neuron pathway. 


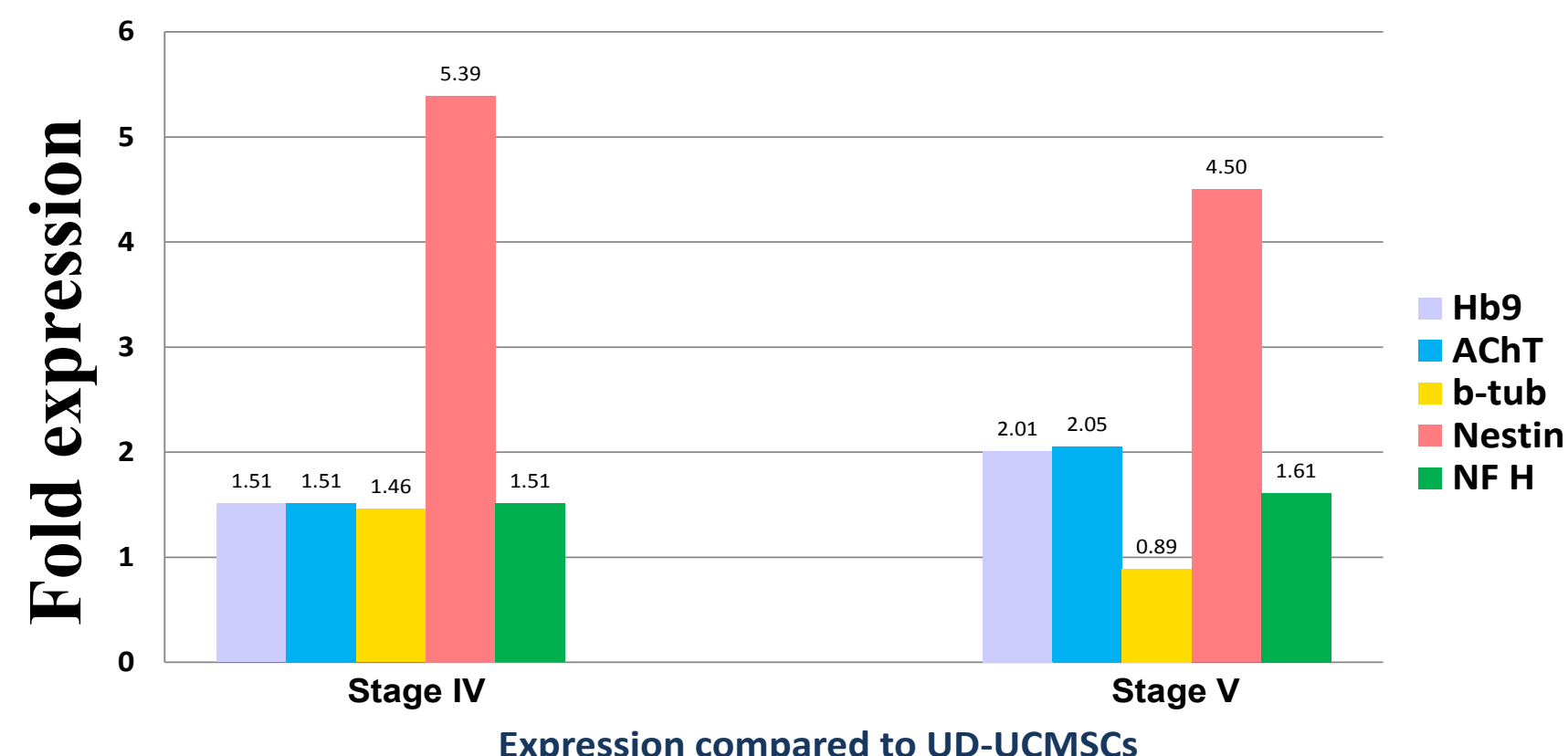

Figure 3: qRT-PCR analysis of neuronal genes expressed by the motor neuron like cells

The fold change at post-induction stages are relative to the undifferentiated MSCs. CT values obtained using ABI real-time machine were analysed using the real-time ABI system SDS 7500 version 1.2.2 software. Expression of various motor neuronal genes such as Nestin, b-tubulin, NF H, Hb9 and AChT during the two stages of differentiation was assessed.

\section{Cellular Characterization}

Flow cytometric analysis of differentiated MSCs: Expression of neuronal proteins in the cells at all the stages was analyzed by flow cytometry. The expression of pluripotent markers such as Oct 4 and Nanog decreased (Figure 4A). In Neurosphere stage, the expression of Pax 6 and Sox 1 showed increased expression (Figure 4B). Also the expression of neuroepithelial (primitive neuronal) markers increased in the Neurosphere stage suggesting that the cells are differentiated towards neuronal lineage. MAP-2 and Islet 1 have shown increased expression in neurosphere stage. But Stage IV showed a decreased expression and expression increased in stage V. Motor Neuron specific markers Olig 2 and Hb9 showed an increased expression in stage V (Figure 4C). Flow cytometric analysis revealed that most of the stage $\mathrm{V}$ differentiated cells were positive for motor neuron marker Acetyl Choline Transferase $73.57 \%$ compared to the stage IV cells where the expression was $11.27 \%$. Stage V shows significant rise in the mature motor neuron marker Acetyl Choline Transferase. These results suggest that the protocols results in efficient derivation of motor neurons and their progenitors from UC-MSCs.

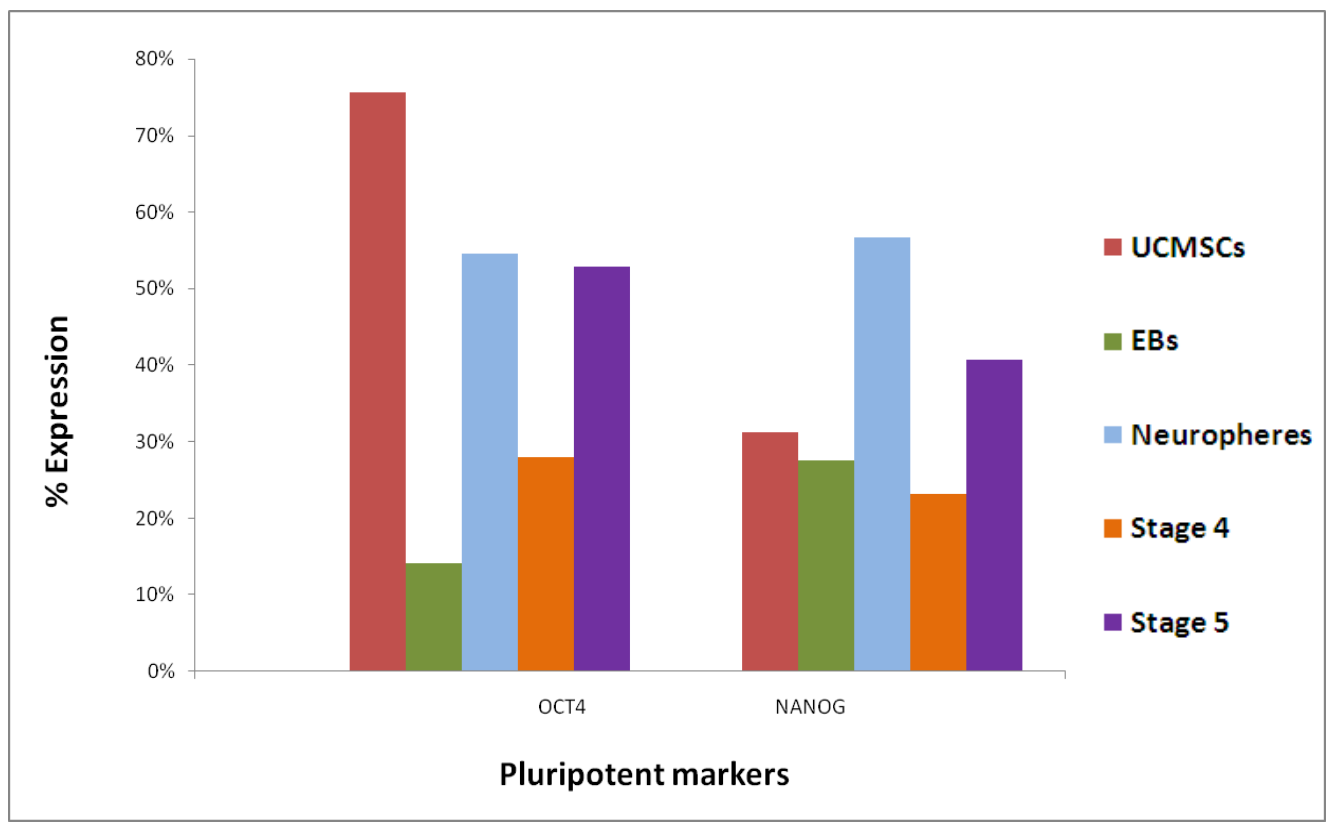

Figure 4 : Flow cytometric analysis: Representative bar graphs of the undifferentiated and differentiated UCMSCs. Cells were characterized for the expression of pluripotent, neuronal and motor neuron specific markers at every stage.

Figure 4 (A): Comparative expression of pluripotent markers at all the stages Expression of Oct-4 was highest in the undifferentiated stage. 


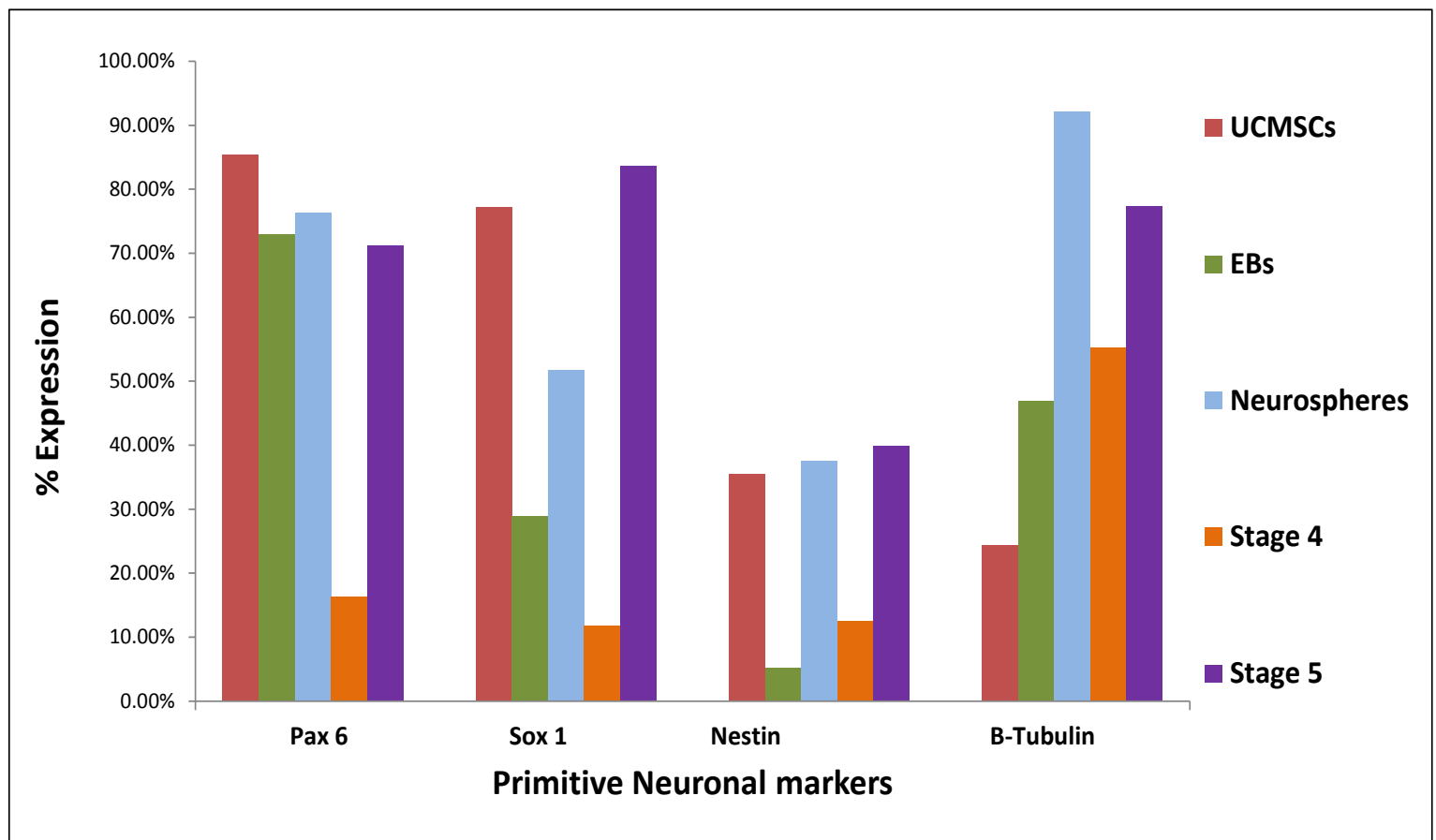

Figure 4 (B): Comparative expression of primitive neuronal/ neural progenitor markers at all the stages. Flow cytometric analysis revealed high expression of neuroepithelial markers such as Pax 6 and Sox 1 in the final stages of differentiation.

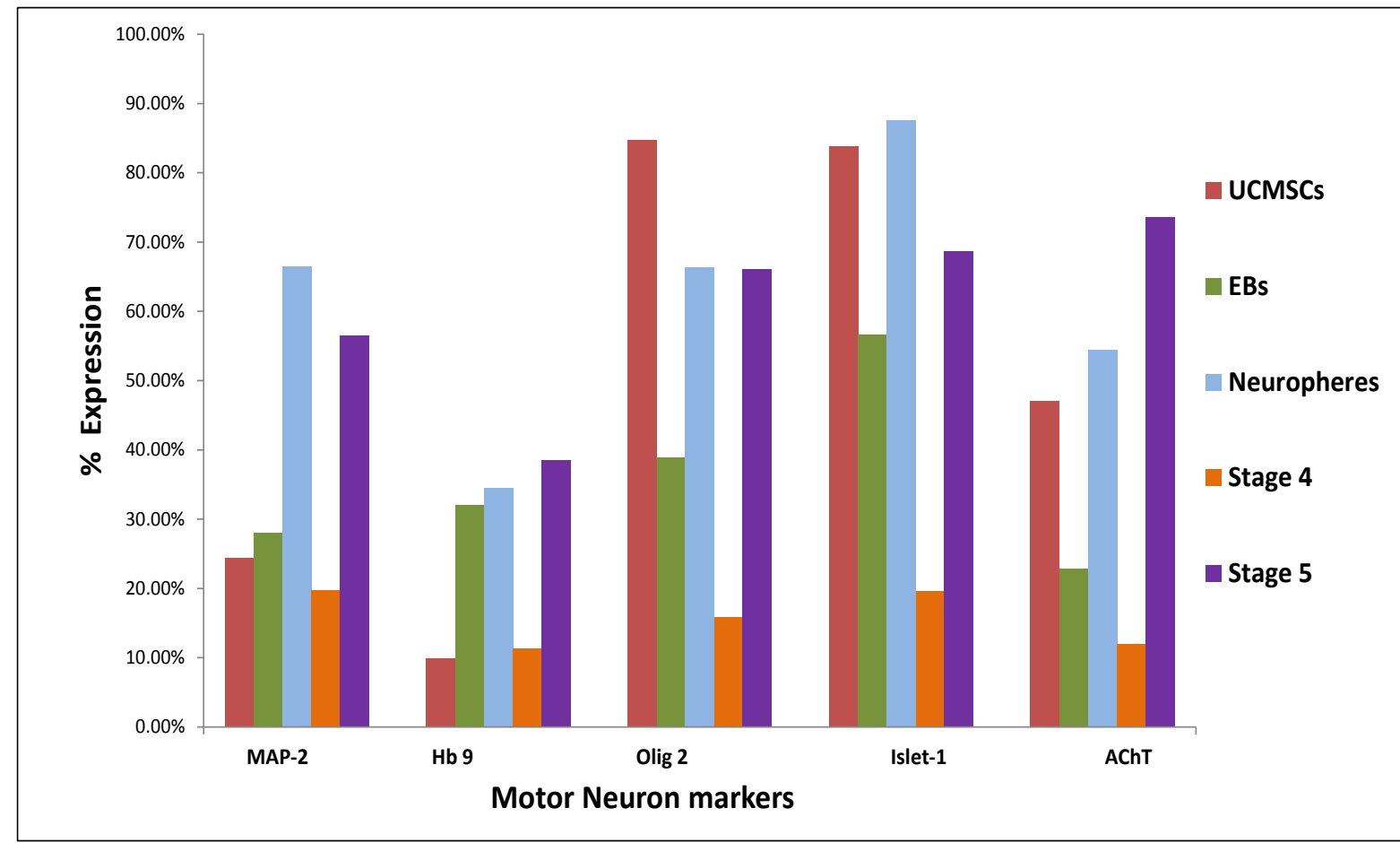

Figure 4 (C): Comparative expression of motor neuron markers ; cells at initial stages weakly expressed motor neuron markers such as $\mathrm{Hb} 9$ and AChT as compared to the differentiated cells in which these markers were upregulated.

\section{Immunocytochemistry}

Stage V differentiated cells were characterized by Immunocytochemistry. Cells were double stained with various markers labeled with different fluorochromes. Immunocytochemical analysis as shown in Figure 5A revealed that stage V cells showed expression of motor neuron markers Olig 2, Hb9 and AChT along with the other neuronal markers like Neu N, Neurofilament, MAP-2 and Islet-1. AChT is expressed by motor neurons secreting neurotransmitter AChT. Olig 2 and Hb9 are transcription factors expressed by motor neurons. Neurofilament (NF) protein is expressed by neuronal cells showing neurofilaments and Neuronal Nuclei (Neu $\mathrm{N}$ ) stains neural nucleus which is seen to be co-stained with DAPI in the Figure 5B. Differentiated cells also showed typical motor neuron morphology in addition to the high expression of motor neuron markers. 

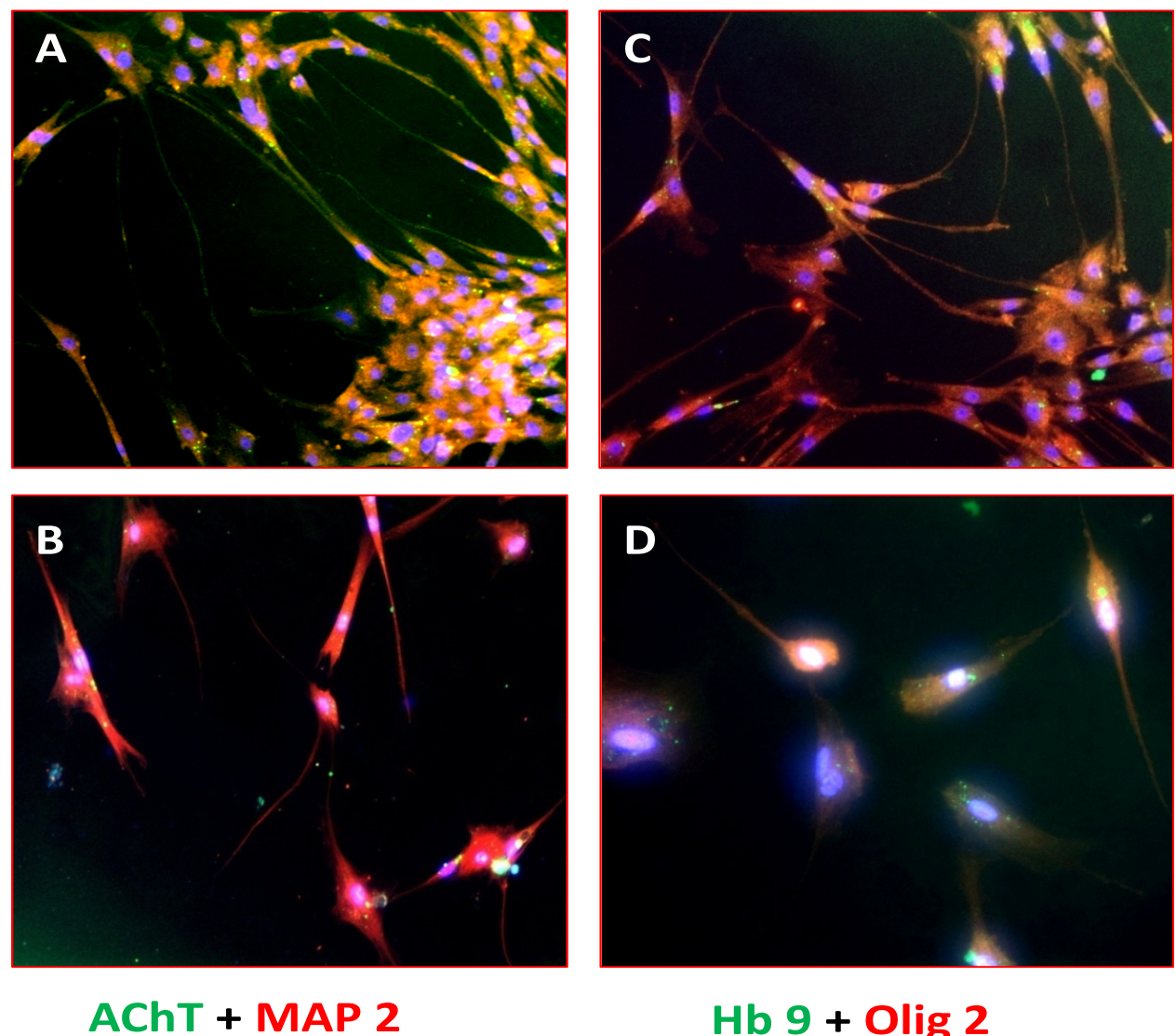

Hb 9 + Olig 2

Figure 5 (A): Immunofluorescence analysis of the differentiated cells (Stage V) using various biomarkers such as A,B: AChT(green) Motor Neurons also stained positively for MAP-2 (red); C,D: Hb9(green) positive motor neurons also stained for Olig 2(red). For the detection of unlabeled antibodies, secondary antibody labeled to Alexa Flour 488 and Alexa Flour 568 was used. The nucleus was stained blue by DAPI. The slides were observed at 10X magnification using a fluorescence microscope.
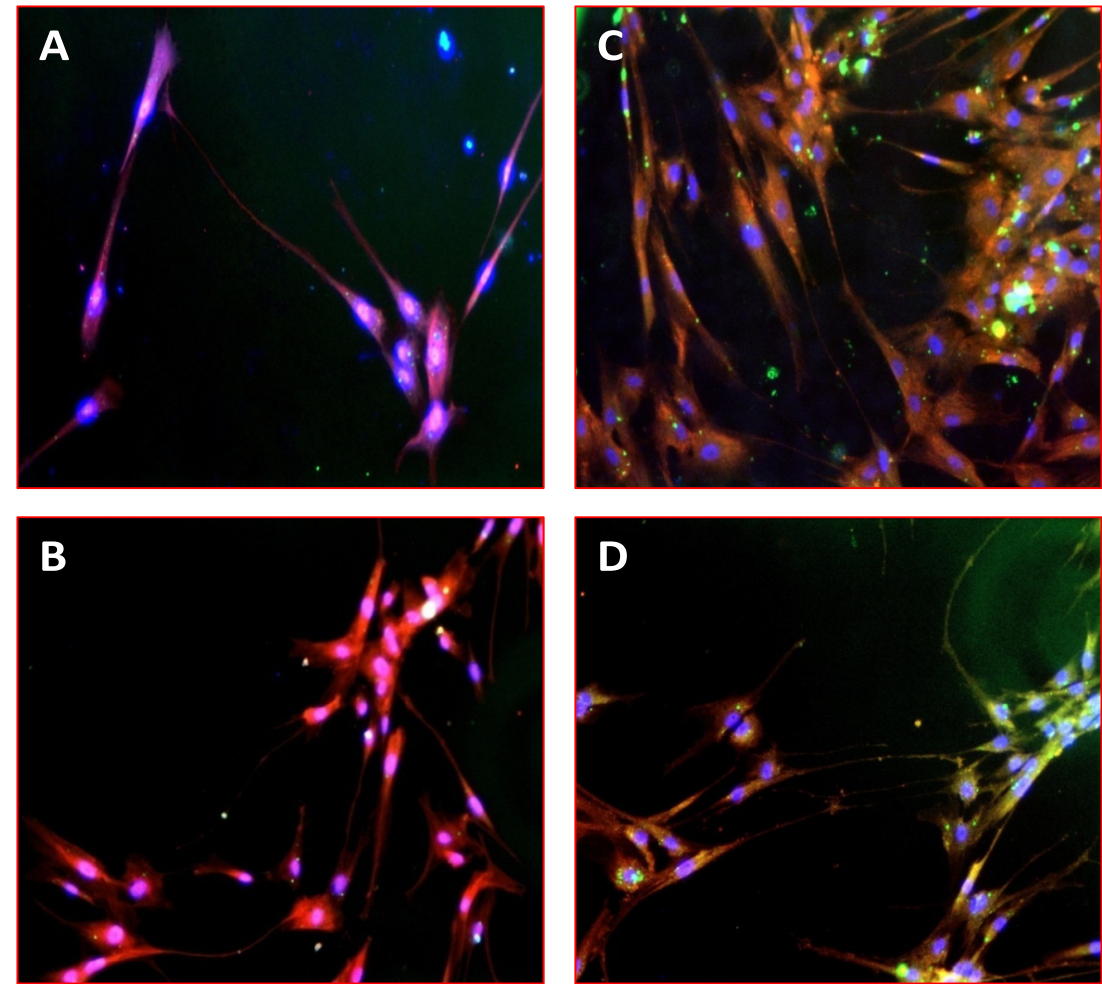

Neu N + NF

\section{$\beta$-tub + Islet-1}

Figure 5 (B): Immunofluorescence analysis of the differentiated cells (Stage V) using various biomarkers such as A,B: Neu N (green) positive motor neurons also stained for NF (red); C,D: $\beta$-tubulin(red) motor neurons also stained postively for Islet-1(green). For the detection of unlabeled antibodies, secondary antibody labeled to Alexa Flour 488 and Alexa Flour 568 was used. The nucleus was stained blue by DAPI. The slides were observed at 10X magnification using a fluorescence microscope. 


\section{Discussion}

Stem cell based replacement strategies show a promising future for the treatment of motor neuron diseases due to their ability to replace lost, damaged or dysfunctional neurons. Embryonic stem cells are pluripotent stem cells derived from embryos and have huge potential in the field of tissue engineering and regenerative medicine as they hold the capacity to produce every type of cell and tissue in the body i.e. differentiation.

Immense research has been carried on efficient differentiation of ESCs to Motor Neurons. MNs derived from ESC could exhibit synaptic communication with muscle cells with an in vitro co-culture culture [11]. Wichterle H. et al. [4] derived motor neurons from hESCs which could form synapses with target muscles. The findings of Shin S., Takazawa T. [12,13] show that human embryonic stem cell derived motor neurons develop characteristics typical of spinal motor neurons and suggest that they are a relevant and useful platform for studying motor neuron development and function and for modeling motor neuron diseases. Despite the therapeutic promise offered by ESCs, due to the ethical issues, they have a limitation in clinical application. There is a risk that allogeneic ES cell-derived cells could be rejected by the host. Although the immunogenicity of the transplant can be contained through the lifelong use of immunosuppressive drugs, they are associated with many various side effects and there is also a risk of teratoma formation [14]. Recently, Su H. [15] demonstrated that human iPSCs derived from mesenchymal cells of the umbilical cord possessed a high yield in neural differentiation. After transplanting in injured musculocutaneous nerve of rats, these cells formed functional connections with the target muscle. Qu Q et al. [1] developed an efficient method differentiation of mature and functional MNs from human pluripotent stem cells.

Adult Stem Cells are multipotent stem cells derived from various adult tissues. MSCs are considered suitable candidates for cellbased therapies owing to their intrinsic capacity to self-renew and differentiate. The ability of human mesenchymal stem cells (MSCs) to home into various organs after systemic administration into animals and their ability to differentiate into various adult cells show that these cells can repair damaged organs. Bone marrow is considered a well-accepted source of MSCs. But MSCs have also been isolated from other tissue sources like adipose tissue, dental pulp, umbilical cord blood, cord matrix derived MSCs. Thus they are ideal candidates for replacement therapies and can be transplanted autologously without the risk of immune rejection. Bone marrow (BM-MSCs) are widely used but the collection of BM-MSCs involves invasive procedure. Umbilical cord is a medical waste and non-controversial source of human MSCs. Due to their high plasticity, low immunogenicity and abundant availability, human UC-MSCs could be ideal for cell therapy to treat the injured nervous system, including degenerative MN diseases [10]. Since there has been increasing attention on the therapeutic approach of stem cell derived motor neurons, MSCs are gaining importance. Park et al. [8] derived functionally active motor neuron cells but from genetically engineered hMSCs. In 2013, Liu X. et al. [9] induced UC-MSCs to motor neuron like cells but with a very low efficiency.

In our present study, we have used umbilical cord tissue MSCs using a cell aggregate culture system for differentiation and characterized these motor neuron like cells at various stages. Molecular studies show that expression of Oct 4 and Nanog was reduced at the later stages of differentiation. Olig 2, $\mathrm{Hb} 9$ and AChT which are motor neuron specific markers showed increased level of expression at final stages as compared to the previous stages. Molecular analysis showed that in the early stages of differentiation (Stage III), the neural progenitor markers like Pax 6 and Sox 1 and primitive neuronal markers Nestin and $\beta$-tubulin are upregulated as compared to the undifferentiated UC-MSCs and EB like aggregates. Also, differentiated cells were expressing high levels of motor neuron markers along with mature motor neuron marker AChT compared to the stage IV cells. Immunofluorescence shows typical motor neuronal morphology in addition to high expression of motor neuron markers. Taken together, our results lead to a novel strategy for MN derivation in vitro and hence would provide tool for cell therapy of MND patients in future (Figure 6).

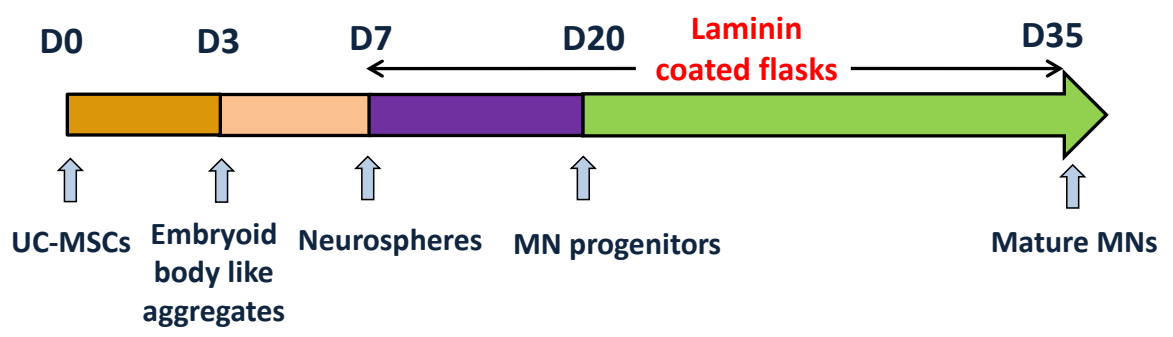

Stage I Stage II Stage III Stage IV Stage V
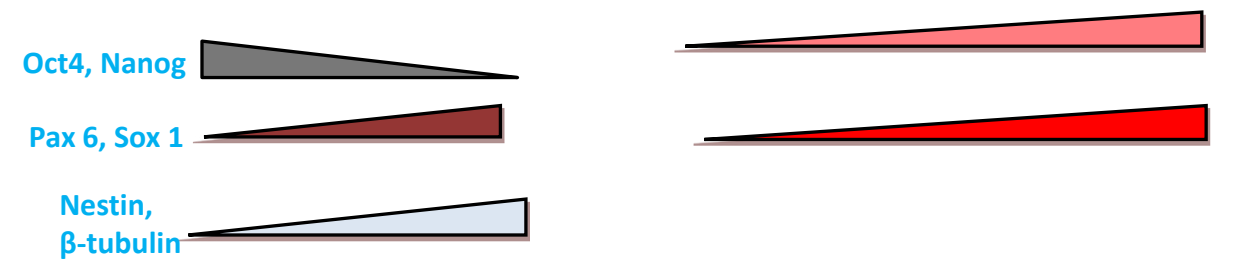

Olig 2, $\mathrm{Hb} 9$

Figure 6 : Summary of the MN derivation from UCMSCs at different stages of differentiation 
As previously described, human ESCs have been widely differentiated to MNs. However, immune rejection and ethical controversy are great hurdles to the clinical application of ESCs in humans. With UC-MSCs, there are ongoing clinical trials and have raised a hope for patients. UC-MSCs are excellent candidates for cellular therapy as they encompass greater differentiation potential and hence can be used for treating patients suffering from various neurodegenerative diseases.

\section{Conclusion}

Many scientists have demonstrated the differentiation of ESCs to motor neurons. Recently there have been reports of differentiation of iPSCs to Motor Neurons. In this study, we have trans-differentiated UC-MSCs to motor neuron like cells upon induction. Hence this is a novel research in the field of regenerative medicine. Upon induction with motor neuron differentiation media, the cells showed motor-neuron like morphology with many cellular processes. The cells were characterized at each stage by molecular characterization using RT-PCR, Real Time PCR and cellular characterization using Flow Cytometry and Immunocytochemistry. Terminally differentiated cells showed significant expression of mature motor neuron marker AChT (73.57\%). Molecular Studies with RT-PCR revealed that the expression of Motor Neuron markers Olig 2, Hb9 and AChT was high in Stage V (differentiated cells). The cells were also characterized using Immunofluorescence for marker proteins like $\beta$-tubulin, NeuN, Neurofilament, MAP-2, Islet-1 Olig2, HB9, and Acetyl Choline Transferase which revealed high expression of markers along with motor neuron morphology. The growth factor cocktail used in the protocol yielded high number of differentiated cells with characteristic morphology and biomarker expression. In the present study, we have differentiated and characterized Motor neuron like cells derived from Umbilical cord tissue mesenchymal stem cells (UC-MSCs) and hence derived a protocol for efficient differentiation of adult stem cells to motor neurons. This protocol can now be routinely used for the derivation of Motor Neurons and its scale up for preclinical and clinical purposes. Based on this study, an in vivo study will be carried out, in which the cells will be tested for its safety and efficacy in an animal model and will enable us to understand the functional role of these cells in treating MND.

\section{Acknowledgement}

The authors acknowledge Reliance Life Sciences Pvt. Ltd (www.rellife.com), for providing the infrastructure and financial support to work on this project.

\section{References}

1. Qu Q, Li D, Louis KR, Li X, Yang H, et al. (2014) High-efficiency motor neuron differentiation from human pluripotent stem cells and the function of Islet-1. Nature Commu 5: 3449.

2. Leigh PN, Ray-Chaudhuri K (1994) Motor neuron disease. J Neurol Neurosurg Psychiatry 57: 886-96.

3. MND association.

4. Wichterle H, Lieberam I, Porter J, Jessell T (2002) Directed Differentiation of Embryonic Stem Cells into Motor Neurons. Cell 110: 385-97.

5. Hu BY, Zhang SC (2010) Directed differentiation of neural-stem cells and subtype-specific neurons from hESCs. Methods Mol Biol 636: 123-37.

6. Rossi SL, Nistor G, Wyatt T, Yin HZ, Poole AJ, et al. (2010) Histological and Functional Benefit Following Transplantation of Motor Neuron Progenitors to the Injured Rat Spinal Cord. PLoS ONE 5: el1852.

7. Baksh D, Song L, Tuan RS (2004) Adult mesenchymal stem cells: characterization, differentiation, and application in cell and gene therapy. J Cell Mol Med 8: 301-16.

8. Park HW, Cho JS, Park CK, Jung SJ, Park CH, et al. (2012) Directed Induction of Functional Motor Neuron-Like Cells from Genetically Engineered Human Mesenchymal Stem Cells. PLoS ONE 7: e35244.

9. Liu X, Li D, Jiang D, Fang Y (2013) Acetylcholine secretion by motor neuron-like cells from umbilical cord mesenchymal stem cells. Neural Regen Res 8: 2086-92. 10. Shetty P, Thakur AM, Viswanathan C (2013) Dopaminergic cells, derived from a high efficiency differentiation protocol from umbilical cord derived mesenchymal stem cells, alleviate symptoms in a Parkinson's disease rodent model. Cell Biol Int 37: 167-80.

11. Umbach JA, Adams KL, Gundersen CB, Novitch BG (2012) Functional Neuromuscular Junctions Formed by Embryonic Stem Cell-Derived Motor Neurons. PLoS One 7: e36049.

12. Shin S, Dalton S, Stice SL (2005) Human Motor Neuron Differentiation from Human Embryonic Stem Cells. Stem Cells Dev 14: 1-4.

13. Takazawa T, Croft GF, Amoroso MW, Studer L, Wichterle H, et al. (2012) Maturation of Spinal Motor Neurons Derived from Human Embryonic Stem Cells. PLoS ONE 7: e40154.

14. Guido de Wert, Mummery C (2003) Human embryonic stem cells: research, ethics and policy. Human Reproduction 18: 672-82.

15. Su H, Wang L, Cai J, Yuan Q, Wu W et al. (2013) Transplanted motoneurons derived from human induced pluripotent stem cells form functional connections with target muscle. Stem Cell Res 11: 529-39. 


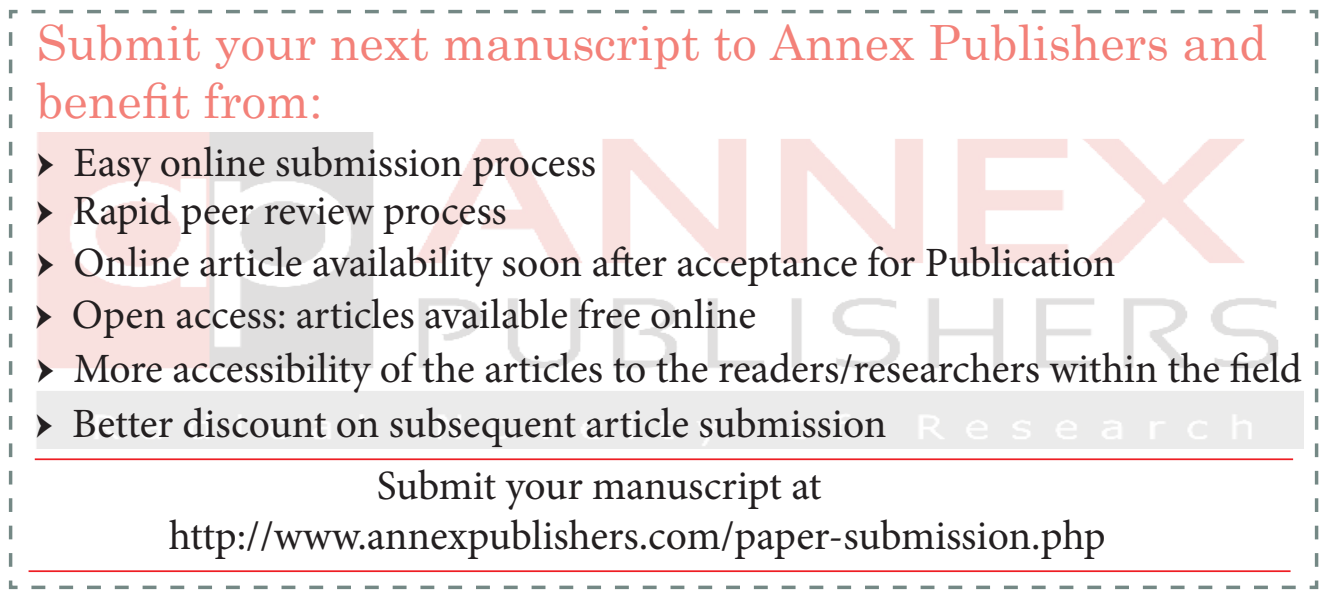

